\title{
Visfatin/PBEF/Nampt induces EMMPRIN and MMP-9 production in macrophages via the NAMPT-MAPK (p38, ERK1/2)-NF-кB signaling pathway
}

\author{
YUQI FAN $^{1 *}$, SHU MENG $^{2 *}$, YUE WANG ${ }^{1}$, JIATIAN CAO $^{1}$ and CHANGQIAN WANG ${ }^{1}$ \\ ${ }^{1}$ Department of Cardiology, Shanghai Ninth People's Hospital, Shanghai Jiaotong University \\ School of Medicine, Shanghai 200011; ${ }^{2}$ Department of Cardiology, Xinhua Hospital, \\ Shanghai Jiaotong University School of Medicine, Shanghai 200092, P.R. China
}

Received November 24, 2010; Accepted January 14, 2011

DOI: $10.3892 / \mathrm{ijmm} .2011 .621$

\begin{abstract}
The adipocytokine visfatin is closely associated with metabolic disorders. This study explored the effects of visfatin on macrophage-induced inflammation in atheroma. The ability of visfatin to enhance extracellular matrix metalloproteinase inducer (EMMPRIN) expression, matrix metalloproteinase-9 (MMP-9) production and enzymatic activity in THP-1 derived macrophages as well as the mechanisms involved were investigated. EMMPRIN and MMP-9 mRNA levels were investigated by RT-PCR. EMMPRIN and MMP-9 protein levels, nuclear factor (NF)- $\kappa$ B - p65 protein levels, peroxisome proliferator-activated receptor $\gamma(\operatorname{PPAR} \gamma)$ protein levels, and mitogen-activated protein kinase (MAPK) phosphorylation were determined by Western blotting. MMP-9 enzymatic activity was assayed by gelatin zymography. Visfatin (50-400 $\mathrm{ng} / \mathrm{ml}$ ) induced EMMPRIN and MMP-9 depending on the dosage used. Visfatin elicited the activation of $N F-\kappa B$ and MAPK (p38, ERK1/2). Exogenous nicotinamide mononucleotide (NMN), the product of nicotinamide phosphoribosyltransferase (NAMPT) activity, mimicked the effects of visfatin on MAPK (p38, ERK1/2)-NF- $\kappa$ B activation and EMMPRIN/MMP-9 induction. Using the p38 inhibitor, SB203580, the ERK1/2 inhibitor PD98059, the NF- $\kappa$ B inhibitor, pyrrolidine dithiocarbamate and the NAMPT inhibitor FK866, we demonstrated that the visfatin pro-inflammatory action was through the NAMPT-MAPK (p38, ERK1/2)$\mathrm{NF}-\kappa \mathrm{B}$ pathway. Furthermore, the visfatin pro-inflammatory action was not prevented by insulin receptor blockade or by a
\end{abstract}

Correspondence to: Dr Changqian Wang, Department of Cardiology, Shanghai Ninth People's Hospital, Shanghai Jiaotong University School of Medicine, 639 Zhizaoju Road, Shanghai 200011, P.R. China

E-mail: xinhuawcq@gmail.com

${ }^{*}$ Contributed equally

Key words: atherosclerosis, visfatin, inflammation, cell signaling
PPAR $\gamma$ agonist. Visfatin did not modulate PPAR $\gamma$ expression. Retinoid $X$ receptor (RXR) agonist suppressed the effects of visfatin on EMMPRIN/MMP-9, NF- $\kappa$ B, but not on MAPK activation. In conclusion, we have demonstrated that visfatin enhances atheroma inflammation through the NAMPTMAPK (p38, ERK1/2)-NF-кB-EMMPRIN/MMP-9 pathway, a key feature of atherosclerotic diseases linked to metabolic disorders.

\section{Introduction}

Visfatin, initially described as pre-B-cell colony-enhancing factor (PBEF) or nicotinamide phosphoribosyltransferase (NAMPT), is a novel identified adipocytokine. It is preferentially released by visceral fat (1). Enhanced circulating visfatin/PBEF/Nampt (for concision, mentioned as visfatin in the following text) levels have been reported in patients affected by metabolic disorders, such as diabetes mellitus, obesity, or the metabolic syndrome $(2,3)$. The pathophysiological relevance of visfatin remains poorly understood, especially in obesity-associated cardiovascular diseases. Recently, it is hypothesized that visfatin might directly promote atheroma inflammation, therefore representing a link between metabolic disorders and acute coronary syndrome (ACS, including acute myocardial infarction and unstable angina). It has previously been reported that enhanced circulating levels of visfatin are positively associated not only with increased plasma levels of inflammatory markers (2), but also with vascular damage and endothelial dysfunction $(4,5)$.

However, the ability of visfatin to exert direct pro-inflammatory effects on macrophages, which is the main source of inflammatory factors in atheroma, remains largely unknown. To address this question, THP-1 cells were cultured and differentiated into macrophages to investigate the ability of visfatin to up-regulate extracellular matrix metalloproteinase inducer (EMMPRIN) and matrix metalloproteinase-9 (MMP-9) expression, which play the most important roles in atherosclerotic plaque destabilization $(6,7)$. The study further aimed to identify the mechanism mediating the proinflammatory action of visfatin in macrophages. 


\section{Materials and methods}

Reagents. RPMI-1640 medium and fetal bovine serum (FBS) were from Gibco. Phorbol-12-myristate 13-acetate (PMA) was from Calbiochem (San Diego, CA). Human recombinant visfatin was from Axxora (Nottingham, UK). The reagents 9-cis retinoic acid [9-cis, a natural ligand of retinoid $\mathrm{X}$ receptor (RXR)], dimethyl sulfoxide, nicotinamide mononucleotide (NMN), and pyrrolidine dithiocarbamate (PDTC, an NF- $\kappa$ B inhibitor) were from Sigma-Aldrich (St. Louis, MO). SB203580 (SB, a specific p38 inhibitor), PD98059 (PD, a specific MEK/ERK inhibitor), and SP600125 (SP, a specific JNK inhibitor) were from Tocris Bioscience (Bristol, UK). Hydroxy-2-naphthalenylmethylphosphonic acid tris-acetoxymethyl ester (HNMPA, an insulin receptor inhibitor) was from Calbiochem (La Jolla, CA, USA). The reagents 15-deoxy-12, 14-prostaglandin J2 [PGJ2, a natural ligand for peroxisome proliferator-activated receptor $\gamma($ PPAR $\gamma$ )], pioglitazone (piog, a synthetic ligand for PPAR $\gamma$ ), and FK866 (FK, an NAMPT activity inhibitor) were from Cayman Chemical (Ann Arbor, MI). The anti-p38, ERK1/2, and JNK (both phosphorylated and non-phosphorylated) antibodies were from Cell Signaling Technologies (Danvers, MA). The EMMPRIN monoclonal antibody was from Zymed (San Francisco, CA). The MMP-9 and the $\beta$-actin monoclonal antibodies were from Abcam (Cambridge, UK). The PPAR $\gamma$ monoclonal and the NF-kB p65 polyclonal antibody were from Santa Cruz (Santa Cruz, CA). The goat anti-rabbit secondary antibody (No. A-21109) and the goat anti-mouse secondary antibody (No. A-21058) were from Invitrogen (Carlsbad, CA). All other chemicals were of the highest purity available commercially.

Cell culture and treatment. The human monocyte cell line, THP-1 (American Type Culture Collection, Rockville, MD), was used. THP-1 cells can be differentiated into macrophages that mimic human macrophage activity and function, particularly in atherosclerotic lesions. Thus, this cell line has been widely used in studies of macrophage function in atherosclerosis $(8,9)$. Cells were maintained at a density of $10^{6}$ cells $/ \mathrm{ml}$ in RPMI-1640 medium supplemented with $10 \%$ FBS and $10 \mathrm{mM}$ HEPES (Sigma) under 5\% $\mathrm{CO}_{2}$ at $37^{\circ} \mathrm{C}$ in an incubator. Cells were then cultured in PMA $(100 \mathrm{nM})$ in 6-well plates for $48 \mathrm{~h}$ to promote differentiation into macrophages. After three washes with sterile PBS, cells were sub-cultured in fresh medium.

After being starved with $0.3 \%$ FBS for $12 \mathrm{~h}$, macrophage cells were stimulated with different concentrations of visfatin $(0,50,100,200,400 \mathrm{ng} / \mathrm{ml})$ or NMN $(100 \mu \mathrm{mol} / \mathrm{l})(10)$. Cells were also treated with visfatin $(200 \mathrm{ng} / \mathrm{ml})$ for different times (0, 5, 10, 15, 30, 60 min). Inhibitors (SB, PD, JNK, PDTC, HNMPA, and FK866) or ligands (piog, PGJ2, and 9-cis) were added $1 \mathrm{~h}$ prior to incubation with visfatin $(200 \mathrm{ng} / \mathrm{ml})$ for $15 \mathrm{~min}$ or $24 \mathrm{~h}$.

$R N A$ isolation and RT-PCR. Total RNA was extracted from visfatin-treated cells using the Trizol reagent. Total RNA $(2 \mu \mathrm{g})$ was then reverse-transcribed into cDNA using a random primer and the resultant cDNA was amplified by PCR with the following primer: EMMPRIN (163 bp), 5'-TCCTGGGCATCGTGGCT-3' (sense), and 5'-CCTCTGGCGGACGTTCTTG-3' (antisense);
MMP-9 (115 bp), 5'-ACTACTGTGCCTTTGAGTCC-3' (sense), and 5'-AGAATCGCCAGTACTTCCC-3' (antisense); $\beta$-actin (249 bp), 5'-CATGTACGTTGCTATCCAGGC-3' (sense), and 5'-CTCCTTAATGTCACGCACGAT-3' (antisense). All gels were detected by the Tanon-4100 digital Gis image system (Beijing, China). Densitometric analysis was performed with Quantity One (Bio-Rad) to scan the signals.

Preparation of cytoplasmic and nuclear extracts. Macrophages were rinsed in cold PBS and lysed in a solution containing $0.6 \%$ Nonidet P-40, $10 \mathrm{mM} \mathrm{KCl}, 10 \mathrm{mM}$ HEPES, $0.1 \mathrm{mM}$ EDTA (all products from Sigma Aldrich), and Complete ${ }^{\mathrm{TM}}$ MiniEDTA-free protease inhibitor cocktail (Roche Diagnostics). After centrifugation (30 sec, 2,000 x g), supernatants were incubated on ice for $5 \mathrm{~min}$. Nuclei were precipitated by centrifugation $\left(4^{\circ} \mathrm{C}, 3 \mathrm{~min}, 15,000 \mathrm{x} \mathrm{g}\right)$, supernatants collected as cytosolic extracts, and the nuclei resuspended in a solution of 10\% glycerol, $20 \mathrm{mM}$ HEPES, $400 \mathrm{mM} \mathrm{NaCl}, 1 \mathrm{mM}$ EDTA and Complete Mini-EDTA-free protease inhibitor cocktail. The mixture was incubated on ice for $1 \mathrm{~h}$, the supernatant was collected after centrifugation for $5 \mathrm{~min}$ at 15,000 $\mathrm{xg}$, and saved as nuclear extracts.

Western blotting. Protein concentrations were measured by the BCA Protein Assay (Pierce, Rockford). The protein extracts were then denatured and the solubilized proteins $(20 \mu \mathrm{g})$ were electrophoresed on $10 \%$ polyacrylamide SDS gels. The proteins were subsequently transferred onto polyvinylidene difluoride membranes (Millipore, MA) and then blocked with TBS with $0.05 \%$ Tween-20 and 5\% skim milk for $1 \mathrm{~h}$ at room temperature, followed by probing with the primary antibody overnight, and then with the secondary antibody labeled with the far-red-fluorescent dye, Alexa Fluor 680. All signals were detected by Odyssey (Li-Cor, USA). Densitometric analysis was performed with the Quantity One software.

Zymography. MMP activity was determined by SDS-polyacrylamide gel zymography. Briefly, the culture supernatant was collected, loaded onto a $10 \%$ polyacrylamide gel containing $0.1 \%$ gelatin, which was run at $125 \mathrm{~V}$ for $90 \mathrm{~min}$. The gel was renatured in renaturing buffer for $30 \mathrm{~min}$. After equilibration in developing buffer for $30 \mathrm{~min}$, fresh developing buffer was added, and the gelatin-containing gel was allowed to develop overnight at $37^{\circ} \mathrm{C}$. The gelatin gel was stained with $0.5 \%$ Coomassie blue in a buffer consisting of $10 \%$ acetic acid, $50 \%$ methanol, and $40 \%$ distilled water before being destained in the same buffer for $30 \mathrm{~min}$ to visualize the zymogen bands. An image of each gel (after drying) was scanned and the zymogen bands were quantified by using densitometry. Results are expressed in arbitrary densitometry units.

Statistical analysis. Data comparisons were performed by the Student's t-test with the SPSS 13.0 software. Results are expressed as the mean $\pm \mathrm{SD}$. P-values $<0.05$ were considered significant. All experiments were repeated at least 3 times.

\section{Results}

Visfatin enhances EMMPRIN and MMP-9 expression as well as MMP-9 enzymatic activity in macrophages. In macrophages 
A

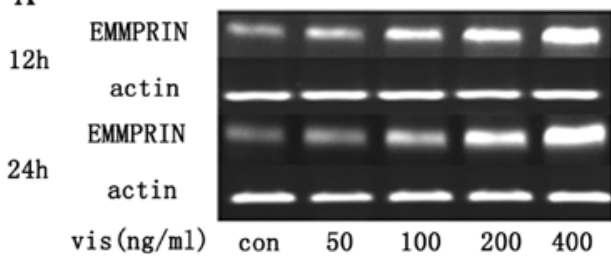

C
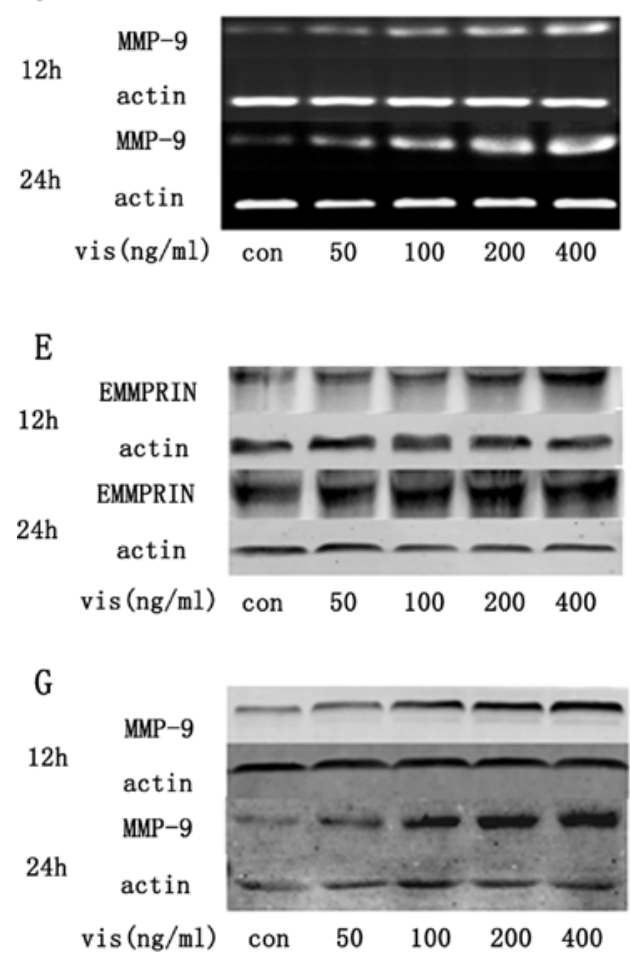

I

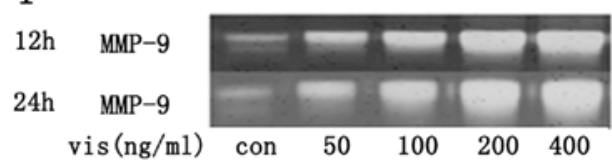

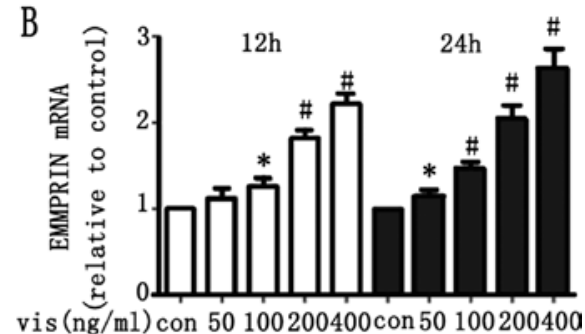

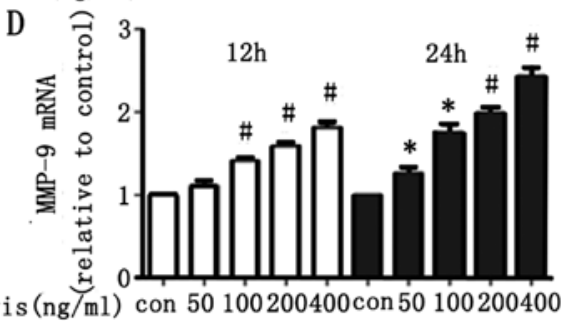

F

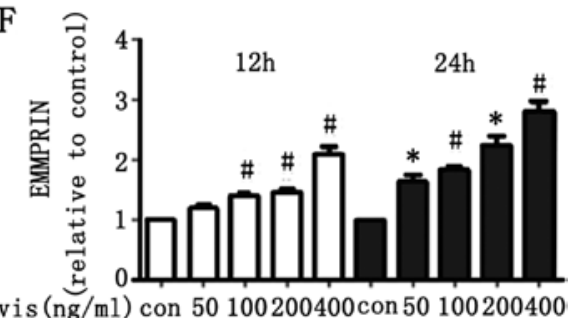

$\mathrm{H}$
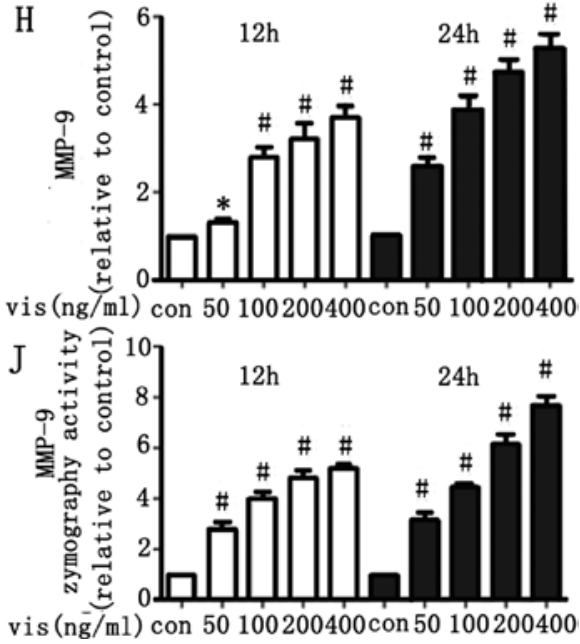

Figure 1. Visfatin (vis) induces EMMPRIN and MMP-9 expression in macrophages. Effects of visfatin on (A) EMMPRIN mRNA, (C) MMP-9 mRNA, (E) EMMPRIN protein production, (G) MMP-9 protein production and (I) on MMP-9 enzymatic activity. (B, D, F, H and J) Respective densitometric analysis measurements. Values are the mean $\pm \mathrm{SD}(\mathrm{n}=3)$. Band density of untreated macrophages were defined as control and set to 1 . ${ }^{*} \mathrm{P}<0.05$; ${ }^{\#} \mathrm{P}<0.01$.

exposed to visfatin $(0,50,100,200$, and $400 \mathrm{ng} / \mathrm{ml})$ for 12 or $24 \mathrm{~h}$, a concentration-dependent increase in the mRNA and protein levels of EMMPRIN and MMP-9 was observed over time.

With a threshold concentration of $100 \mathrm{ng} / \mathrm{ml}$ (after $12 \mathrm{~h}$ ) or $50 \mathrm{ng} / \mathrm{ml}$ (after $24 \mathrm{~h}$ ), visfatin induced a significant dosedependent increase in the mRNA expression of EMMPRIN and its downstream inflammatory factor MMP-9, with a maximum response at $400 \mathrm{ng} / \mathrm{ml}$ (Fig. 1A-D). There was a peak increase of 2.63-fold in EMMPRIN and a peak increase of 2.46-fold in MMP-9 after $24 \mathrm{~h}$.

The protein expression of EMMPRIN and MMP-9 following 12 or $24 \mathrm{~h}$ visfatin treatments $(0,50,100,200$, and $400 \mathrm{ng} / \mathrm{ml}$ ) were measured by Western blot analysis. The results were similar to those observed at the mRNA level
(Fig. 1E-H). Peak increases of 2.79-fold in EMMPRIN and of 5.29-fold in MMP-9 were observed after $24 \mathrm{~h}$.

Furthermore, the enzymatic activity of MMP-9 was assessed by gelatin zymography. Visfatin dose-dependently up-regulated MMP-9 enzymatic activity over time (Fig. 1I and J). There was a peak increase of 7.7-fold after $24 \mathrm{~h}$.

Visfatin pro-inflammatory action requires the MAPK

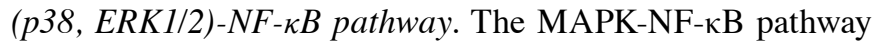
plays an important role in atheroma destabilization as it regulates the production of inflammatory factors. To ascertain whether visfatin signals via this pathway in macrophages, macrophages were treated with visfatin and compared with the control subjects (untreated). In macrophages, visfatin $(200 \mathrm{ng} / \mathrm{ml})$ elicited a gradual increase in nuclear NF- $\mathrm{B}$ p65 
A

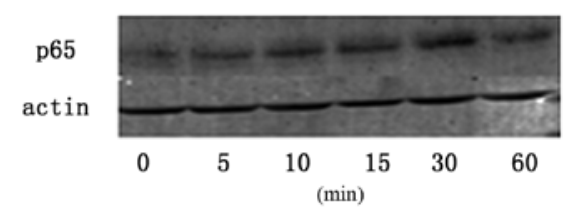

C

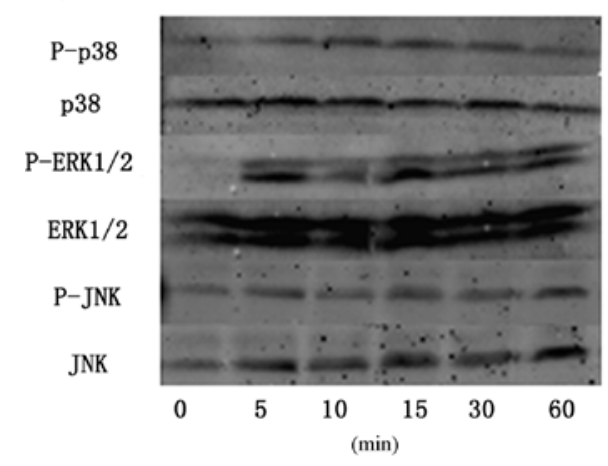

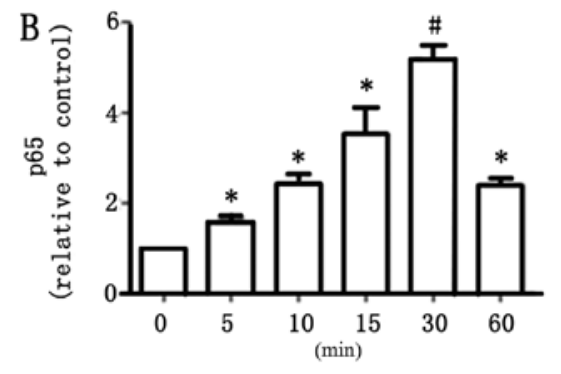

D

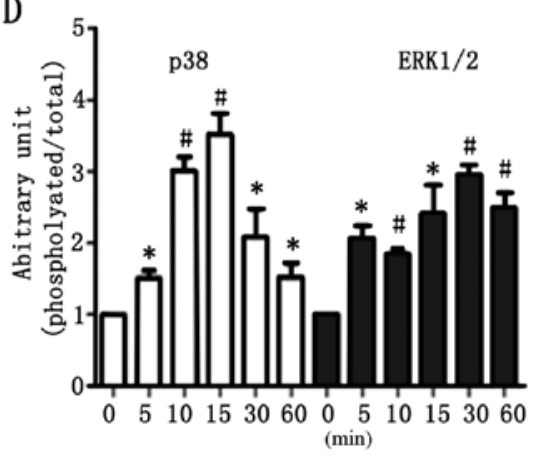

Figure 2. Visfatin activates MAPK (p38, ERK1/2) and NF- $\kappa$ B. Effect of visfatin on (A) nuclear NF- $\mathrm{B}$ p65 levels and (C) MAPK activation. (B and D) Respective densitometric analysis measurements. Values are the mean \pm SD $(n=3)$. The band density of untreated macrophages were defined as control and set to 1 . ${ }^{*} \mathrm{P}<0.05$ and ${ }^{\#} \mathrm{P}<0.01$, compared to control.

from 5-30 min, when the maximal activation was attained. At $60 \mathrm{~min}$, the NF- $\mathrm{B}$ p 65 level was about $50 \%$ of that achieved at $30 \mathrm{~min}$, although it remained enhanced compared to the basal levels (Fig. 2A and B). In addition, visfatin significantly activated ERK1/2 and p38 MAPK (Fig. 2C and D). Visfatin increased the phosphorylation of both p42 and p44 (ERK1/2). It attained a maximum at $30 \mathrm{~min}$ and decreased thereafter. The adipocytokine also increased p38 phosphorylation. It attained a maximum response at $15 \mathrm{~min}$ and then gradually decreased. Visfatin had no effect on JNK phosphorylation (Fig. 2C and D).

The implications of both NF-кB and MAPK (p38, ERK1/2) in visfatin-mediated EMMPRIN and MMP-9 inductions in macrophages were then assessed. Macrophages were pre-treated with inhibitors of p38 (SB, $10 \mu \mathrm{M})$, ERK1/2 (PD, $50 \mu \mathrm{M})$, JNK (SP, $10 \mu \mathrm{M})$, or of NF- $\kappa \mathrm{B}$ (PDTC, $100 \mu \mathrm{M})$ for $1 \mathrm{~h}$, prior to visfatin treatment $(200 \mathrm{ng} / \mathrm{ml})$ for $24 \mathrm{~h}$. The co-incubation of visfatin $(200 \mathrm{ng} / \mathrm{ml})$ with SB or PD significantly suppressed the inductions of EMMPRIN and MMP-9 by the adipocytokine. PDTC completely blocked visfatin pro-inflammatory action (Fig. 3A-F). Furthermore, $\mathrm{SB}$ and $\mathrm{PD}$ attenuated $\mathrm{NF}-\kappa \mathrm{B}$ activation triggered by visfatin (Fig. 3G and H), highlighting the role of MAPK (p38, ERK1/2) in NF- $\mathrm{NB}$ activation by visfatin. Based on these findings, it can be concluded that visfatin exerts pro-inflammatory effects on macrophage via the MAPK (p38, ERK1/2)-NF- $\mathrm{B}$ pathway.

NAMPT enzymatic activity mediates the pro-inflammatory action of visfatin in macrophages. Since it has been reported that visfatin may exert its cellular actions through its intrinsic NAMPT enzymatic activity (11), we explored whether NMN, the product of NAMPT activity and a precursor of NAD (12), could mimic the pro-inflammatory effect of visfatin in macrophages. Exogenous NMN (100 $\mu \mathrm{mol} / \mathrm{l})$, activated
MAPK (p38, ERK1/2)-NF- $\mathrm{B}$ to a similar extent with visfatin (200 ng/ml) (Fig. 4A and B). NMN (100 $\mu \mathrm{mol} / \mathrm{l})$ also triggered EMMPRIN and MMP-9 induction in macrophages, which was not significantly different from that achieved by $200 \mathrm{ng} / \mathrm{ml}$ visfatin (Fig. 4C and D).

The impact of the inhibition of the NAMPT activity on the pro-inflammatory action of visfatin was next assessed. The activation of MAPK ( $\mathrm{p} 38$, ERK1/2)-NF- $\kappa$ B elicited by visfatin $(200 \mathrm{ng} / \mathrm{ml})$ in macrophages was completely abolished by co-incubation with the NAMPT activity inhibitor FK866 (10 nmol/l) (13-15) (Fig. 4E and F). Similarly, FK866 (10 nmol/l) blocked the induction of EMMPRIN and MMP-9 by $200 \mathrm{ng} / \mathrm{ml}$ visfatin (Fig. 4G and H). Based on these results, it can be concluded that NAMPT enzymatic activity mediates the pro-inflammatory action of visfatin in macrophages. It is worth noting that the NAMPT substrate nicotinamide is contained in the culture medium of macrophages (RPMI-1640).

The pro-inflammatory action of visfatin in macrophages is not mediated by the insulin receptor. Visfatin was initially proposed as an insulin mimetic (1). Therefore, whether the pro-inflammatory action of visfatin in macrophages is mediated by the insulin receptor was explored. For this purpose, macrophages were pre-treated with HNMPA (100 $\mu \mathrm{mol} / \mathrm{l})$, an inhibitor of insulin receptor signaling (16), for $1 \mathrm{~h}$ prior to co-stimulation with visfatin $(200 \mathrm{ng} / \mathrm{ml})$. EMMPRIN induction was then examined. Pre-incubation of macrophages for $1 \mathrm{~h}$ with HNMPA did not affect the NF- $\mathrm{B}$ activation by visfatin (200 ng/ml) after $15 \mathrm{~min}$ (Fig. 4I and J).

A ligand of $R X R$, not PPAR, attenuates visfatin inductions of EMMPRIN and MMP-9 by modulating NF- $\kappa B$ activation. To gain further insight into the mechanisms of the visfatin proinflammatory actions in macrophages, the role of the nuclear 
A

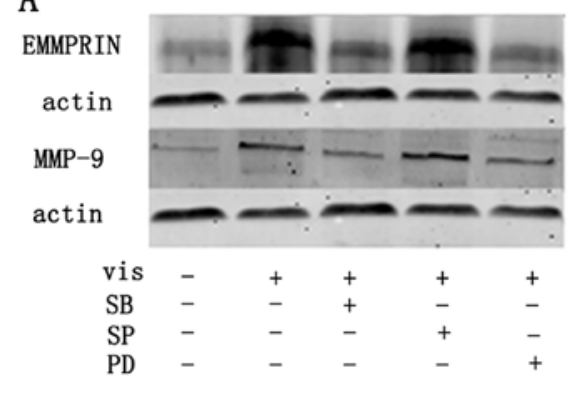

C

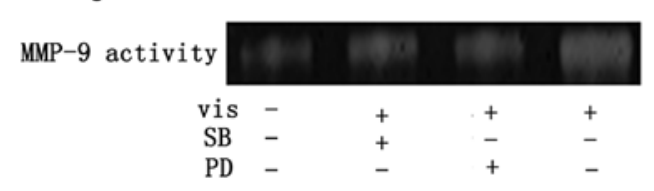

E

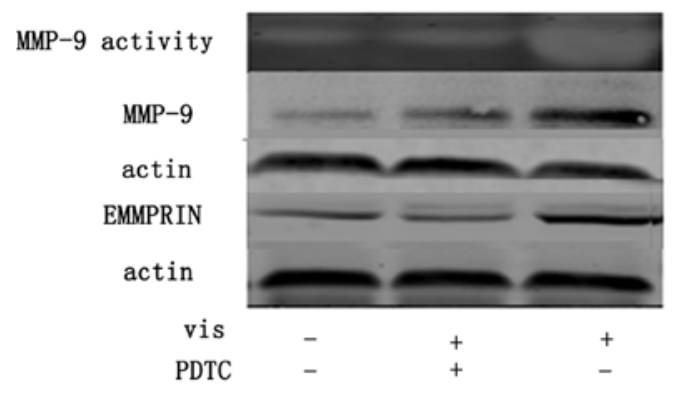

G

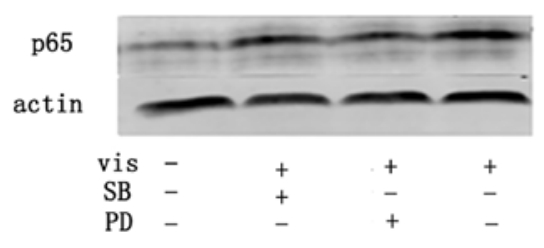

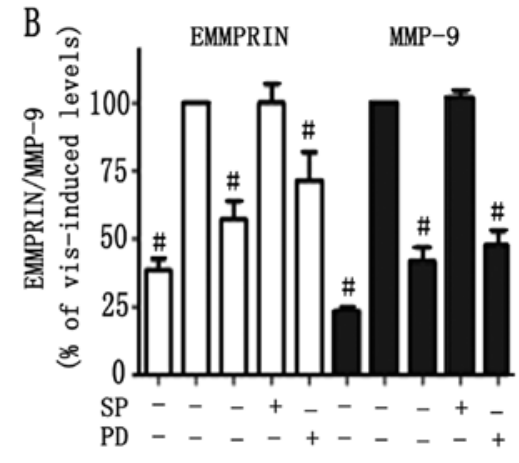

D

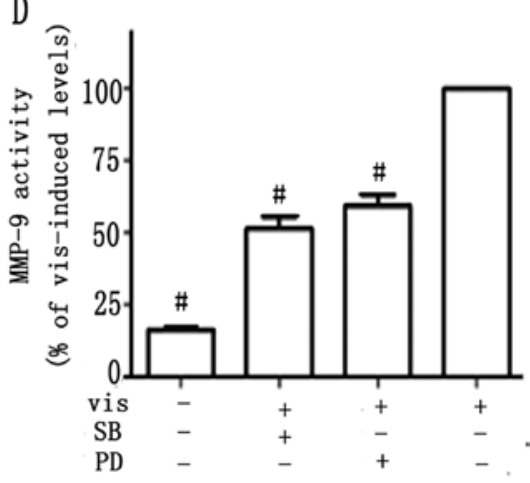

$\mathrm{F}$

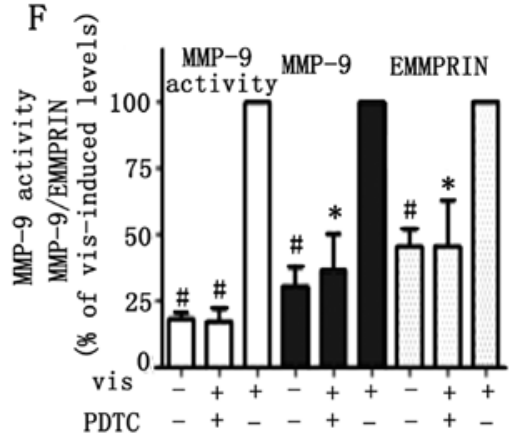

$\mathrm{H}$

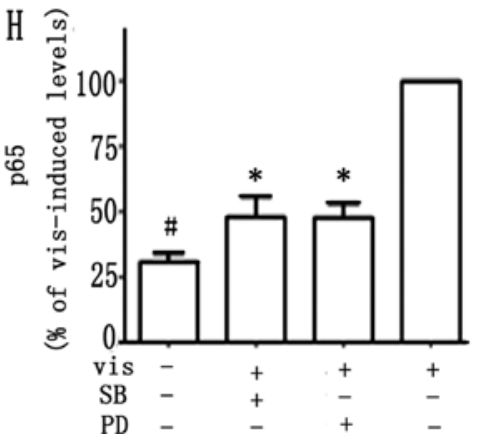

Figure 3. Inhibitors of MAPK (p38, ERK1/2) and NF- $\kappa B$ block the pro-inflammatory action of visfatin (vis). Inhibitors of MAPK (p38, ERK1/2) also suppress NF- $\mathrm{kB}$ activation by visfatin stimulation. Cells were pre-treated with the $\mathrm{p} 38$ MAPK inhibitor, SB, the ERK1/2 MAPK inhibitor PD, the JNK MAPK inhibitor, $\mathrm{SP}$, or the NF-kB inhibitor, PDTC for $1 \mathrm{~h}$, prior to incubation with visfatin for $24 \mathrm{~h}$ or $15 \mathrm{~min}$. (A) Effect of SB, PD, or SP on the expression of EMMPRIN and MMP-9 by visfatin stimulation. (C) Effect of SB and PD on MMP-9 activity by visfatin stimulation. (E) Effect of PDTC on visfatin pro-inflammatory action. (G) Effect of SB and PD on nuclear NF-kB p65 levels. Respective densitometric analysis measurements (B, D, F and H). Values are the mean \pm SD (n=3). The band density of macrophages treated with visfatin $(200 \mathrm{ng} / \mathrm{ml})$ alone was set to 1 . ${ }^{*} \mathrm{P}<0.05$ and ${ }^{\#} \mathrm{P}<0.01$, compared to macrophages treated with visfatin alone.

receptors, RXR and PPAR $\gamma$, were analyzed. For this purpose, the expression of PPAR $\gamma$ in macrophage stimulated with visfatin for $24 \mathrm{~h}$ was assayed by Western blotting. Visfatin did not modulate PPAR $\gamma$ expression (Fig. 5A and B). Cells were then pre-treated with the PPAR $\gamma$ natural ligand PGJ2 $(10 \mu \mathrm{M})$, the synthetic ligand piog $(20 \mu \mathrm{M})$, or the RXR ligand 9-cis $\left(10^{-7} \mathrm{M}\right)$ for $1 \mathrm{~h}$, prior to visfatin treatment $(200 \mathrm{ng} / \mathrm{ml})$ for $24 \mathrm{~h}$. It is notable that 9 -cis attenuated visfatin inductions of EMMPRIN and MMP-9, but PGJ2 or piog did not (Fig. 5C-F). Furthermore, 9-cis attenuated the visfatin-induced activation of NF-kB, but not of MAPK (p38, ERK1/2) (Fig. 5G-J). Based on these results, it can be inferred that RXR is involved in the 
A

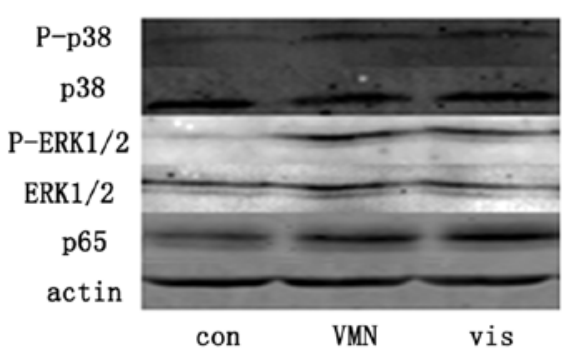

C

MMP-9 activity

MMP-9

actin

EMMPRIN

actin

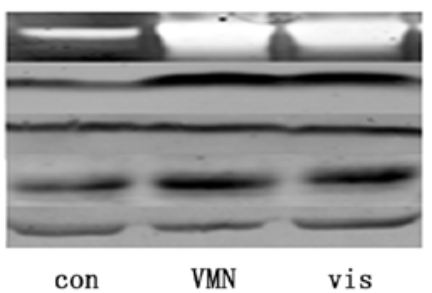

E

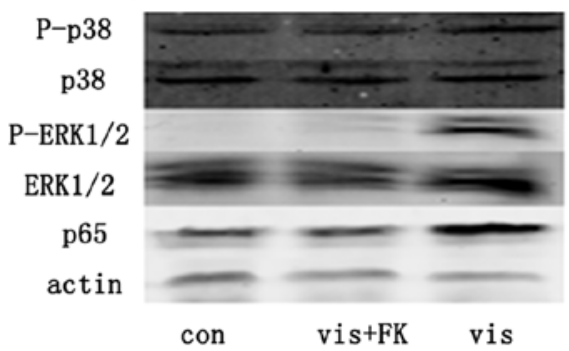

G

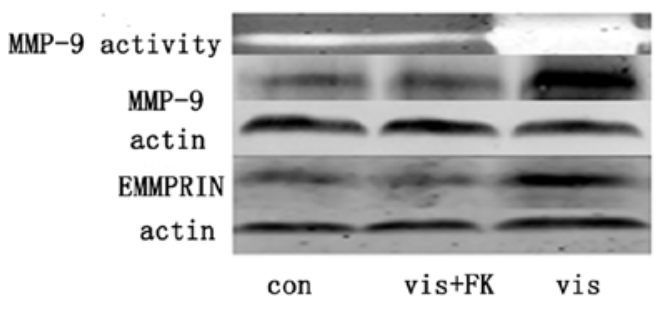

EMMPRIN

\section{I}

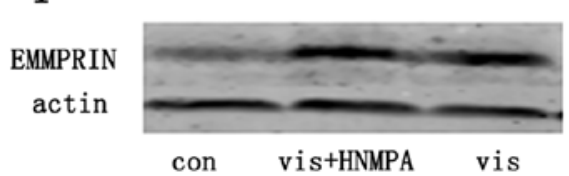

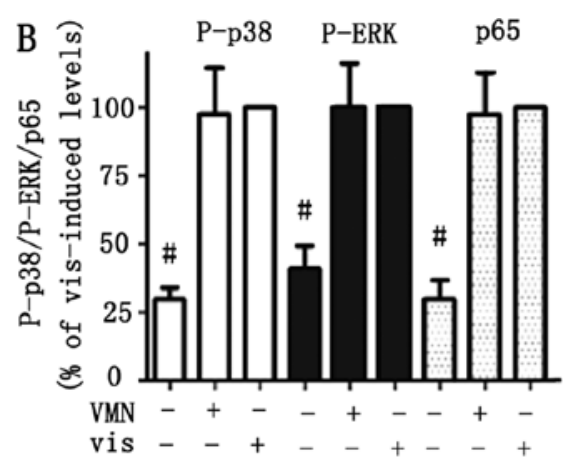

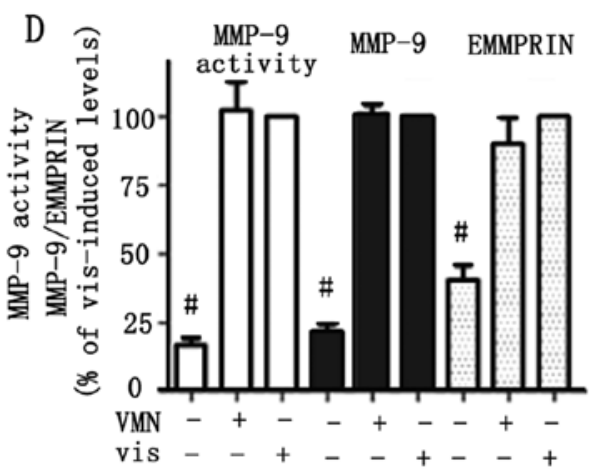

$\mathrm{F}$
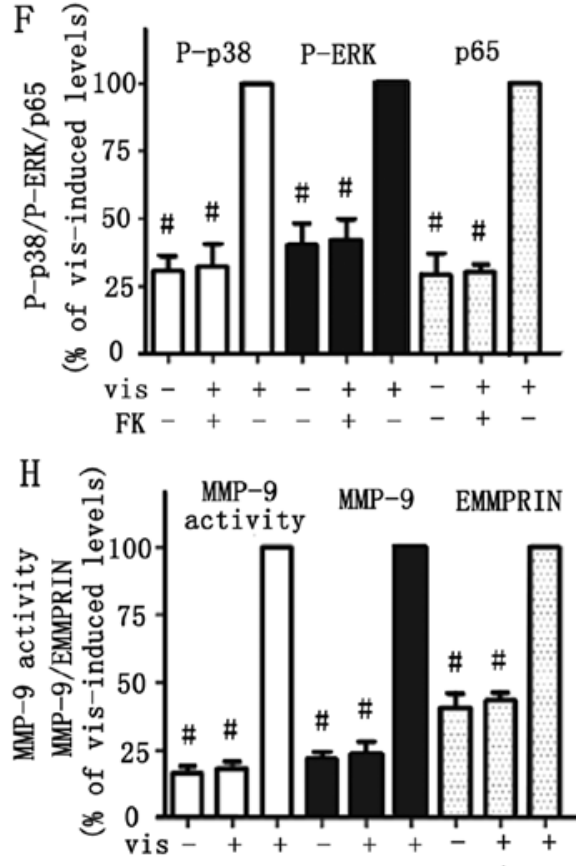

FK -

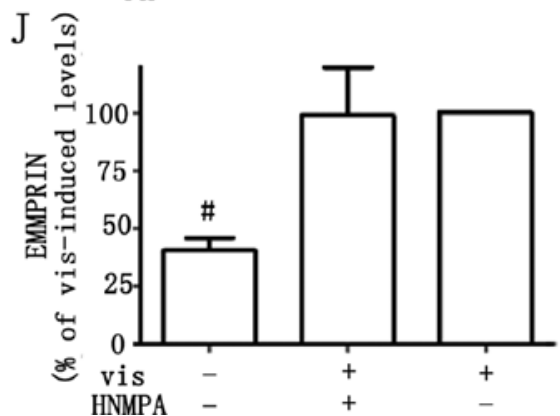

Figure 4. NAMPT activity, but not insulin receptor, is required for the visfatin pro-inflammatory action. (A) Effect of NMN on the activation of MAPK

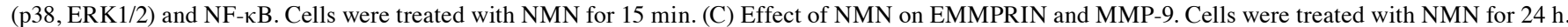
(E) Effect of the NAMPT inhibitor, FK, on the activation of MAPK (p38, ERK1/2) and NF-кB by visfatin stimulation. Cells were pre-treated with FK for $1 \mathrm{~h}$, prior to incubation with visfatin for $15 \mathrm{~min}$. (G) Effect of the NAMPT inhibitor, FK, on the induction of EMMPRIN and MMP-9 by visfatin stimulation. Cells were pre-treated with FK for $1 \mathrm{~h}$, prior to incubation with visfatin for $24 \mathrm{~h}$. (I) Effect of HNMPA on NF- $\mathrm{B}$ activation by visfatin stimulation. (B, D, F, H and J) Respective densitometric analysis results. Values are the mean \pm SD ( $n=3)$. Band density of macrophages treated with visfatin (200 ng/ml) alone was set to 1 . ${ }^{*} \mathrm{P}<0.05$, and ${ }^{\#} \mathrm{P}<0.01$, compared with macrophages treated with visfatin alone. 
A

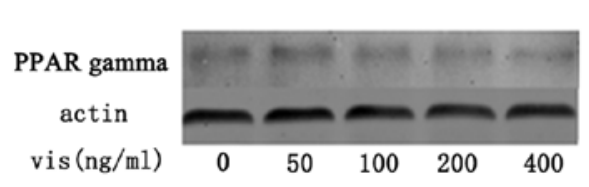

C

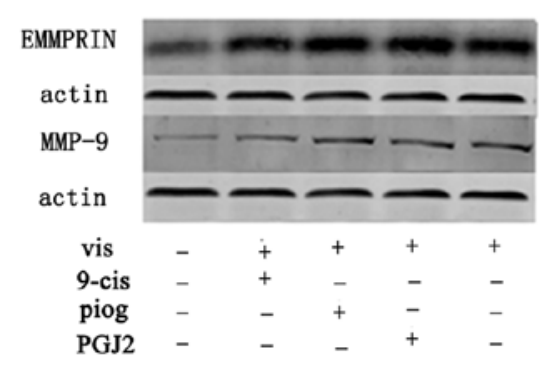

E
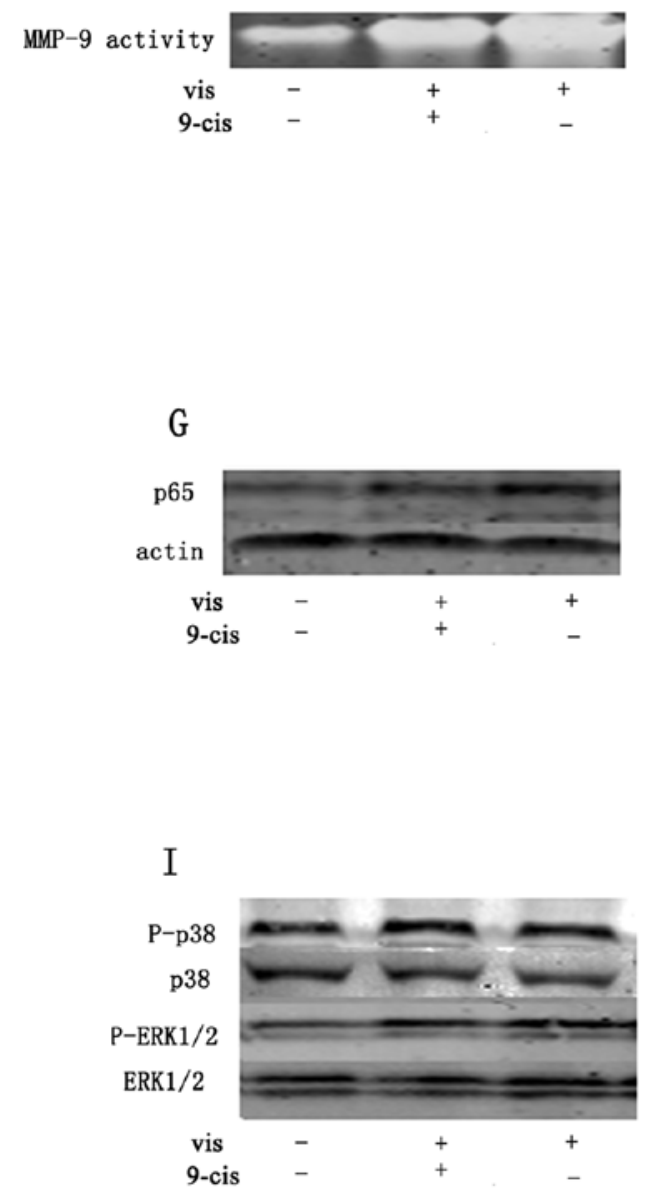
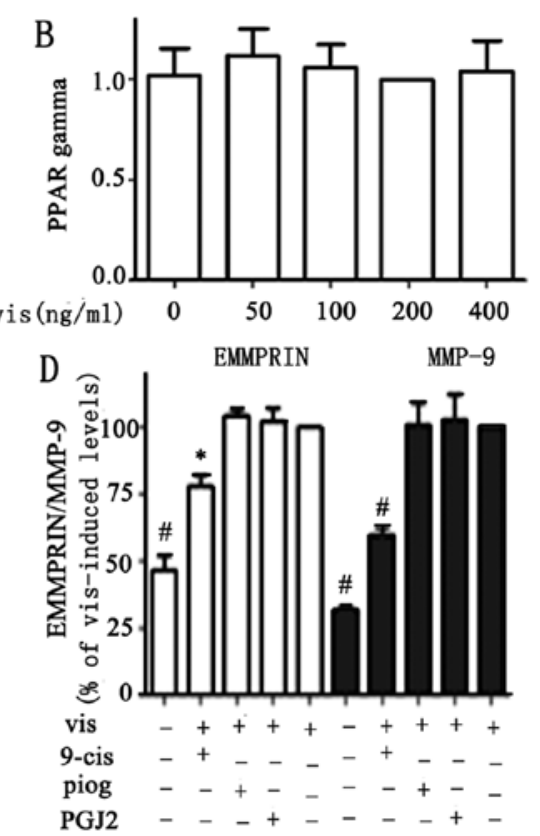

F
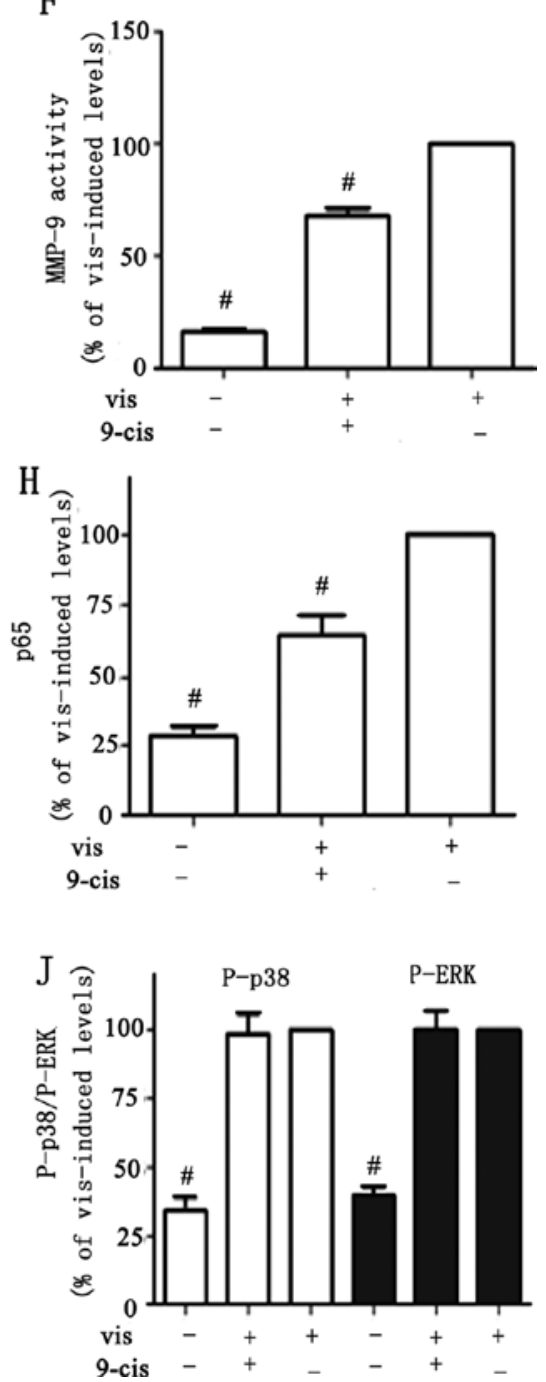

Figure 5. A ligand of RXR, but not of PPAR $\gamma$, attenuates the pro-inflammatory action of visfatin (vis) by modulating NF- $\mathrm{B}$ activation. (A) Effect of visfatin on PPAR $\gamma$ expression. (C) Effect of 9-cis, piog, or PGJ2 on EMMPRIN and MMP-9 production by visfatin stimulation. (E) Effect of 9-cis on MMP-9 activity. (G) Effect of 9-cis on nuclear NF-kB p65 levels. Macrophages were pre-treated with 9-cis for $1 \mathrm{~h}$, prior to incubation with visfatin for 15 min. (I) Effect of 9-cis on MAPK (p38, ERK1/2) activation. Macrophages were pre-treated with 9-cis for $1 \mathrm{~h}$, prior to incubation with visfatin for 15 min. (B, D, F, H and J) Respective densitometric analysis results. Values are mean \pm SD ( $\mathrm{n}=3)$. Band density of macrophages treated with visfatin (200 ng/ml) alone was set to 1 . ${ }^{*} \mathrm{P}<0.05$ and ${ }^{\#} \mathrm{P}<0.01$, compared with macrophages treated with visfatin $(200 \mathrm{ng} / \mathrm{ml})$ alone. 
pro-inflammatory action of visfatin by modulating $\mathrm{NF}-\kappa \mathrm{B}$ activation.

\section{Discussion}

The ability of visfatin to exert direct pro-inflammatory effects on macrophages was investigated. This study reports for the first time that visfatin dose-dependently increased EMMPRIN and MMP-9 production as well as MMP-9 enzymatic activity in THP-1-derived macrophages. This pro-inflammatory action was through the NAMPT-MAPK (p38, ERK1/2)-NF- $\kappa$ B pathway. The nuclear receptor RXR is believed to be involved in visfatin's pro-inflammatory action by modulating $\mathrm{NF}-\kappa \mathrm{B}$ activation.

The inflammatory status plays key roles in undermining atherosclerotic plaque stability (7,17-19), which leads to lifethreatening ACS. Metabolic disorders are closely associated with atherosclerosis inflammation (20). The pro-inflammatory effect of visfatin, a novel identified adipocytokine, has been suggested. Enhanced visfatin content has been reported in human unstable carotid and coronary atherosclerotic plaques (16) as well as in the adipose tissue of patients with coronary artery disease compared with the individuals in the control group (21). In monocytes, visfatin has been reported to enhance MMP-9, tumor necrosis factor- $\alpha$, and interleukin-8 expression (16). In endothelial cells, visfatin has ben shown to up-regulate the monocyte chemoattractant protein-1 and MMP-9 production (22-24). In smooth muscle cells, visfatin has been found to induce production of the pro-inflammatory factor, iNOS (10). This study demonstrates herein that visfatin induces EMMPRIN and MMP-9 in macrophages. Both macrophage mRNA expression and protein production of EMMPRIN and MMP were enhanced by visfatin, demonstrating visfatin-induced up-regulation at both the transcriptional and translational level. Furthermore, visfatin also increased MMP-9 enzymatic activity. It is worth noting that the pro-inflammatory action of visfatin in macrophages was observed beginning at a concentration of $50 \mathrm{ng} / \mathrm{ml}$, a level that was reported in obese or type 2 diabetic patients, whereas the corresponding healthy subjects showed lower levels $(2,3)$. Thus, our findings highlight visfatin's pro-inflammatory action as a link between atherosclerotic disease and metabolic disorders.

The MAPK-NF- $\kappa$ B pathway has been proven to be associated with visfatin's action. It has been demonstrated that visfatin up-regulates iNOS via the ERK1/2-NF- $\mathrm{B}$ pathway in smooth muscle cells (10). Liu et al have reported that p38 and ERK1/2 phosphorylation is required in the monocyte chemotactic protein-1 and interleukin- 6 inductions in endothelial cells (25). One of the aims of this study was to elucidate the role of the MAPK-NF- $\kappa \mathrm{B}$ pathway in visfatin inductions of EMMPRIN and MMP-9 in macrophages. Visfatin was found to phosphorylate both ERK1/2 and p38 MAPK. Furthermore, p38 and ERK1/2 inhibitor, respectively, blocked visfatin inductions of EMMPRIN and MMP-9. These results demonstrate the role of MAPK (p38, ERK1/2) in the action of visfatin. Next, the role of NF- $\kappa \mathrm{B}$ was investigated. In this study, the nuclear p65 level was chosen to reflect the degree of $\mathrm{NF}-\kappa \mathrm{B}$ activation. In the inactivated status, dimeric complexes of $N F-\kappa B$ p50/p65 are held in the cytoplasm. When $N F-\kappa B$ is activated, these complexes translocate into the nucleus and regulate gene transcription (26). Therefore, nuclear $\mathrm{NF}-\kappa \mathrm{B}$ p65 levels have been widely accepted to represent the degree of NF- $\kappa \mathrm{B}$ activation. This study found that nuclear p65 levels were up-regulated by visfatin over time. Moreover, inhibitors of p38 and ERK1/2 suppressed the visfatin-induced activation of $\mathrm{NF}-\kappa \mathrm{B}$. The $\mathrm{NF}-\kappa \mathrm{B}$ inhibitor significantly blocked visfatin induction of inflammatory factors. Thus, consistent with aforementioned studies, it can be concluded that the MAPK (p38, ERK1/2)-NF- $\mathrm{B}$ pathway is required for the pro-inflammatory action of visfatin.

One of the major findings in this study is that NAMPT action is required for visfatin's pro-inflammatory action. NAMPT converts nicotinamide to NMN, which is then transformed into NAD by nicotinamide/nicotinic acid mononucleotide adenylyltransferase (12). NAMPT enzymatic activity plays an important role in visfatin's action. Revollo et al (11) have demonstrated that visfatin regulates insulin secretion in pancreatic $\beta$-cells through its intrinsic NAMPT enzymatic activity. Wang et al (27) have reported that visfatin stimulates vascular smooth muscle cell proliferation via the NMN-mediated ERK1/2 and p38 signaling. In a recent study, Romacho et al (10) have shown that NAMPT activity is involved in visfatin's pro-inflammatory action in smooth muscle cells. Our findings are in line with these reports. In this study, exogenous MNM mimicked the effects of visfatin on EMMPRIN, MMP-9, and MAPK (p38, ERK1/2)-NF- $\kappa$ B activation. This stimulatory action of visfatin was abolished upon co-incubation with the NAMPT activity inhibitor, FK866. Taken together, these results strongly suggest that extracellular NMN generation through NAMPT activity is on the basis of the pro-inflammatory activity of visfatin in macrophages.

Visfatin was initially claimed to be an insulin mimetic acting through the insulin receptor by modulating PPAR $\gamma$ levels $(1,16,28,29)$. Such a statement was later retracted and remains highly controversial (30-32). The results of the present study do not support this statement. Indeed, blocking the activation of the insulin receptor did not interfere with $\mathrm{NF}-\kappa \mathrm{B}$ activation by visfatin in macrophages. Furthermore, the PPAR $\gamma$ agonist PGJ2 failed to block visfatin's pro-inflammatory activity, and PPAR $\gamma$ expression was not modulated by visfatin. This discrepancy could be due to different cell types, cell differentiation stages, visfatin concentrations, and PPAR $\gamma$ agonists.

We suggest that RXR is involved in visfatin activity by regulating $\mathrm{NF}-\kappa \mathrm{B}$. RXR is a novel identified nuclear receptor associated with atherosclerosis (33). We found that the RXR agonist significantly suppressed not only visfatin's action on EMMPRIN and MMP-9, but NF- $\mathrm{BB}$ activation. This is in line with other reports. Razny et al have demonstrated that NF- $\kappa \mathrm{B}$ levels are enhanced in hepatocytes of RXR $\alpha$-deficient mice compared to wild-type mice (33). Na et al have reported that I $\mathrm{B} \beta$ interacts with the RXR and inhibits retinoid-dependent transactivation in lipopolysaccharide-treated cells (34). Despite modulating the NF- $\kappa \mathrm{B}$ activation by visfatin, the RXR ligand did not attenuate p38 and ERK1/2 phosphorylation. This implies that there is a much more complicated mechanism for the RXR-mediated effects on the pro-inflammatory action of visfatin.

In conclusion, visfatin is an adipocytokine that promotes macrophage inflammation through the NAMPT-MAPK 
(p38, ERK1/2)-NF- $\mathrm{B}$ pathway. RXR is believed to be involved in this process by modulating $N F-\kappa B$ activation. Our study highlights the pro-inflammatory action of visfatin in macrophages as a link between atherosclerotic disease and metabolic disorders.

\section{Acknowledgements}

This study was supported by the Fund of Science and Technology Commission of Shanghai Municipality (Grants no.07JC14046,08411966900,09XD1403100 and 09JC1410600) and the Fund of National Natural Science Foundation of China (Grant no. 30971436/C0701).

\section{References}

1. Fukuhara A, Matsuda M, Nishizawa M, et al: Visfatin: a protein secreted by visceral fat that mimics the effects of insulin. Science 307: 426-430, 2005.

2. Chen MP, Chung FM, Chang DM, et al: Elevated plasma level of visfatin/pre-B cell colony-enhancing factor in patients with type 2 diabetes mellitus. J Clin Endocrinol Metab 91: 295-299, 2006.

3. Filippatos TD, Derdemezis CS, Kiortsis DN, Tselepis AD and Elisaf MS: Increased plasma levels of visfatin/pre-B cell colony-enhancing factor in obese and overweight patients with metabolic syndrome. J Endocrinol Invest 30: 323-326, 2007.

4. Takebayashi K, Suetsugu M, Wakabayashi S, Aso Y and Inukai T: Association between plasma visfatin and vascular endothelial function in patients with type 2 diabetes mellitus. Metabolism 56 451-458, 2007.

5. Yilmaz MI, Saglam M, Carrero JJ, et al: Normalization of endothelial dysfunction following renal transplantation is accompanied by a reduction of circulating visfatin/NAMPT. A novel marker of endothelial damage? Clin Transplant 23: 241-248, 2009.

6. Kai H, Ikeda H, Yasukawa H, et al: Peripheral blood levels of matrix metalloproteinases- 2 and -9 are elevated in patients with acute coronary syndromes. J Am Coll Cardiol 32: 368-372, 1998.

7. Schmidt R, Bultmann A, Ungerer M, et al: Extracellular matrix metalloproteinase inducer regulates matrix metalloproteinase activity in cardiovascular cells: implications in acute myocardial infarction. Circulation 113: 834-841, 2006.

8. Ouchi N, Kihara S, Arita Y, et al: Novel modulator for endothelial adhesion molecules: adipocyte-derived plasma protein adiponectin. Circulation 100: 2473-2476, 1999

9. Ohki R, Yamamoto K, Mano H, Lee RT, Ikeda U and Shimada K: Identification of mechanically induced genes in human monocytic cells by DNA microarrays. J Hypertens 20: 685-691, 2002.

10. Romacho T, Azcutia V, Vazquez-Bella M, et al: Extracellular $\mathrm{PBEF} / \mathrm{NAMPT} / \mathrm{visfatin}$ activates pro-inflammatory signalling in human vascular smooth muscle cells through nicotinamide phosphoribosyltransferase activity. Diabetologia 51: 2455-2463, 2009.

11. Revollo JR, Korner A, Mills KF, et al: Nampt/PBEF/Visfatin regulates insulin secretion in beta cells as a systemic NAD biosynthetic enzyme. Cell Metab 6: 363-375, 2007.

12. Formentini L, Moroni F and Chiarugi A: Detection and pharmacological modulation of nicotinamide mononucleotide (NMN) in vitro and in vivo. Biochem Pharmacol 77: 1612-1620, 2009.

13. Billington RA, Genazzani AA, Travelli $\mathrm{C}$ and Condorelli $\mathrm{F}$ : NAD depletion by FK866 induces autophagy. Autophagy 4: 385-387, 2008

14. Van der Veer E, Ho C, O'Neil C, et al: Extension of human cell lifespan by nicotinamide phosphoribosyltransferase. J Biol Chem 282: 10841-10845, 2007.
15. Hasmann $\mathrm{M}$ and Schemainda I: FK866, a highly specific noncompetitive inhibitor of nicotinamide phosphoribosyltransferase, represents a novel mechanism for induction of tumor cell apoptosis. Cancer Res 63: 7436-7442, 2003.

16. Dahl TB, Yndestad A, Skjelland M, et al: Increased expression of visfatin in macrophages of human unstable carotid and coronary atherosclerosis: possible role in inflammation and plaque destabilization. Circulation 115: 972-980, 2007

17. Major TC, Liang L, Lu X, Rosebury W and Bocan TM: Extracellular matrix metalloproteinase inducer (EMMPRIN) is induced upon monocyte differentiation and is expressed in human atheroma. Arterioscler Thromb Vasc Biol 22: 1200-1207, 2002.

18. Vaddi K, Nicolini FA, Mehta P and Mehta JL: Increased secretion of tumor necrosis factor-alpha and interferon-gamma by mononuclear leukocytes in patients with ischemic heart disease. Relevance in superoxide anion generation. Circulation 90: 694-699, 1994.

19. Woo CH, Lim JH and Kim JH: Lipopolysaccharide induces matrix metalloproteinase-9 expression via a mitochondrial reactive oxygen species-p38 kinase-activator protein-1 pathway in Raw 264.7 cells. J Immunol 173: 6973-6980, 2004.

20. Rocha VZ and Libby P: Obesity, inflammation, and atherosclerosis. Nat Rev Cardiol 6: 399-409, 2009.

21. Cheng KH, Chu CS, Lee KT, et al: Adipocytokines and proinflammatory mediators from abdominal and epicardial adipose tissue in patients with coronary artery disease. Int J Obes (Lond) 32: 268-274, 2008

22. Adya R, Tan BK, Chen J and Randeva HS: Nuclear factorkappaB induction by visfatin in human vascular endothelial cells: its role in MMP-2/9 production and activation. Diabetes Care 31: 758-760, 2008.

23. Adya R, Tan BK, Chen J and Randeva HS: Pre-B cell colony enhancing factor (PBEF)/visfatin induces secretion of MCP-1 in human endothelial cells: role in visfatin-induced angiogenesis. Atherosclerosis 205: 113-119, 2009.

24. Adya R, Tan BK, Punn A, Chen J and Randeva HS: Visfatin induces human endothelial VEGF and MMP-2/9 production via MAPK and PI3K/Akt signalling pathways: novel insights into visfatin-induced angiogenesis. Cardiovasc Res 78: 356-365, 2008.

25. Liu SW, Qiao SB, Yuan JS and Liu DQ: Visfatin stimulates production of monocyte chemotactic protein-1 and interleukin-6 in human vein umbilical endothelial cells. Horm Metab Res 41: 281-286, 2009.

26. Joyce D, Albanese C, Steer J, Fu M, Bouzahzah B and Pestell RG: NF-kappaB and cell-cycle regulation: the cyclin connection. Cytokine Growth Factor Rev 12: 73-90, 2001.

27. Wang P, Xu TY, Guan YF, Su DF, Fan GR and Miao CY: Perivascular adipose tissue-derived visfatin is a vascular smooth muscle cell growth factor: role of nicotinamide mononucleotide. Cardiovasc Res 81: 370-380, 2009.

28. Xie H, Tang SY, Luo XH, et al: Insulin-like effects of visfatin on human osteoblasts. Calcif Tissue Int 80: 201-210, 2007.

29. Mayi TH, Duhem C, Copin C, et al: Visfatin is induced by peroxisome proliferator-activated receptor gamma in human macrophages. FEBS J 277: 3308-3320, 2010.

30. Fukuhara A, Matsuda M, Nishizawa M, et al: Retraction. Science 318: 565, 2007.

31. Kim SR, Bae YH, Bae SK, et al: Visfatin enhances ICAM-1 and VCAM-1 expression through ROS-dependent NF-kappaB activation in endothelial cells. Biochim Biophys Acta 1783: 886-895, 2008

32. Li Y, Zhang Y, Dorweiler B, et al: Extracellular Nampt promotes macrophage survival via a nonenzymatic interleukin-6/STAT3 signaling mechanism. J Biol Chem 283: 34833-34843, 2008.

33. Razny U, Wator L, Polus A, et al: Hepatocyte RXR alpha deletion in mice leads to inhibition of angiogenesis. Genes Nutr 4: 69-72, 2009.

34. Na SY, Kim HJ, Lee SK, et al: IkappaBbeta interacts with the retinoid $\mathrm{X}$ receptor and inhibits retinoid-dependent transactivation in lipopolysaccharide-treated cells. J Biol Chem 273: 3212-3215, 1998 